\title{
Characters and Significance of Female Breasts in Relation to Female Sexuailty
}

\section{Original Article}

\author{
Ihab Younis, Osama Hussein Abdelsalam, Ahmed Mohammed Hamed, Doaa Samy \\ Department of Dermatology and andrology, Benha Faculty of Medicine, Benha university, \\ Egypt
}

\begin{abstract}
Purpose: The current study was conducted to obtain information about how a sample of Egyptian women and their husbands look at their own breasts, other women's breasts, and the sexual function of breast during intercourse. Method: Self-report questionnaire, designed by the investigators. It included 303 married women with an age range of 18-48 years. Aim: It aimed to obtain information about demography, characters of participants own breast as well as other women's breasts and also the views of their husbands.

Results: The most common shape was "Full on top breast" with reddish brown areolas. The commonest bra size was medium. Most participants thought that large breasts are uncomfortable and only $(11.55 \%)$ of them would like to have a breast augmentation surgery. "Full on top \& bottom" breast shape was the preferred shape. Most participants said that breast size does affect female attractiveness. A majority of responders said that their husbands were satisfied with the size and shape of their breasts. Participants would like their husbands to stimulate their breasts during coitus orally and manually. The ability to reach orgasm by breast stimulation only during coitus was reported by (15.51\%) of participants. Conclusion: The significance of female breasts as a sexual organ must be emphasized especially to the male partner. Some women can reach orgasm by the mere stimulation of their breasts. The study outlined the importance of oral and manual stimulation of breast during foreplay.
\end{abstract}

Key Words: Breasts, females, sexuality.

Received: 9 January 2017, Accepted: 22 March 2017

Corresponding Author: Osama Abdelsalam, Tel.: 002-01001916062, E-mail: oselfady452003@yahoo.com

ISSN: 2090-6048, June 2017, Vol. 7, No. 2

\section{INTRODUCTION}

Female breasts carry an interesting symbolic and political history. Their status as a source and object of erotic pleasure has varied within and across cultures. Breasts have been supported by bras, and sometimes freed from bras. There was a time when push-up bras seemed ridiculous. Even visible bra straps seemed obscene. Breasts were hidden rather than accentuated. Nowadays, the fashion is quite the opposite: breasts should be 'femininely' and 'sexily' pushed out as much as possible ${ }^{[1]}$.

Some studies have shown that small size breasts are the most preferred by men ${ }^{[2,3]}$, while other studies indicated preferences for medium (or medium to large) size ${ }^{[4,5]}$. Yet, most studies showed that men rather prefer large size ${ }^{[6-8]}$. Such inconsistencies can be partly given by methodological differences between the studies, by cultural variation in preferences; and also by inter-individual differences ${ }^{[4]}$.
Evolutionary anthropologists suggest that men should be attracted to those women whose physical characteristics signal the ability to conceive and deliver offspring ${ }^{[9]}$. Among those physical characteristics theorized to reflect female fecundity and, therefore, to enhance women's physical attractiveness to men is large breast size and low waist to hip ratio. Some studies ${ }^{[10,11]}$ found that women with large breasts and relatively narrow waists have higher fecundity as assessed by precise measurements of daily levels of E2 and progesterone. Women who are characterized by both narrow waists and large breasts have 26\% higher mean E2 and 37\% higher mean mid-cycle E2 levels than other women.

The question now is whether there is a difference between men and women in breast size preference. Prantl and Gruünd ${ }^{[12]}$ conducted a study comparing preferences between men and women in a sample consisting of 16,686 men and 17,329 women aged between 15 and 98 years. A 
clear difference was found with regard to the ideal bust size; $40 \%$ of men but only $25 \%$ of women preferred a large bust. However, this difference was challenged by Tovée and Cornelissen ${ }^{[13]}$ who predicted that women have a very precise and accurate idea of what men find attractive and vice versa. This allows them to judge their own relative value with respect to their peer group and match this value with the value of a prospective mate.

In the present study, a sample of Egyptian married women was questioned to gather information about their views about their own breasts as well as the breasts of other women. Besides, the significance of these views as regards their sexuality.

Most studies regarding the sexual significance of females' breast were concerned with the male preference. Women views of their own breasts (e.g. shape or size) are lacking. This information is important for the sexologists to help proper guidance (for both partners) during counseling.

\section{MATERIAL AND METHODS}

This cross-sectional observational study started after approval of the research ethics committee in Benha Faculty of Medicine. Participating women were recruited from the attendants of the outpatient clinic in Benha University Hospital. Selected women were consenting to participate in the study, can read and write and with no history of surgical intervention to breasts. To test the sexual activity and husbands view, all selected females were married. Women with major breast surgery or surgery to change breast size were excluded.

The tool used in this study was a self-report questionnaire designed by the investigators.

Three hundred and thirty questionnaires were distributed but only 303 usable questionnaires were obtained. Women were asked to fill the questionnaire then put it in a sealed envelope then put it in a basket with other envelopes to keep her identity anonymous.

The questionnaire included questions about the following items:

- Epidemiological data e.g. age, educational level and if exposed to female genital cutting.

- Data about the woman's own breasts and her views about the role of female breast in sexuality.

- Husbands' opinions on the sexual significance of female breast.

\section{Statistical data}

The collected data were summarized in terms of frequencies and percentages. Comparisons between the different study groups were carried out using the Chisquared test $(\chi 2)$ and the Fisher's Exact Test (FET) to compare proportions as appropriate. A $P$-value for the calculated tests statistics was obtained. A $P$-value $<0.05$ was considered statistically significant (S), a $P$-value $<0.001$ was considered statistically highly significant (HS), while a $P$-value $>0.05$ was considered statistically non-significant. The statistical analysis was conducted using STATA/SE for Windows, version 11.2 (STATA Corporation, College Station, Texas).

\section{RESULT}

\section{Demographic data}

All women in the study were married. The age range of the participants ranged from 18 to 48 years most of them in the child bearing period $(94.06 \%)$. Most of the participants $(54.79 \%)$ belonged to the age group 2029 years. More than half of women $(54.46 \%)$ had a university degree (Table1). Participants were asked about their weight, however, no actual weighing was done.

\section{Data of participant's own breasts}

"Full on top breast" was the most common shape of our participants own breasts $(39.93 \%)$. For areolar color, reddish brown was the most common color $(62.7 \%)$. The commonest bra size was medium $(30 \%)$. Most participants $(56.11 \%)$ thought that a large breast size would be uncomfortable. , Most of them (66.67\%) were satisfied with breast size and shape when they were teenagers. Only $(11.55 \%)$ of women would like to have a breast augmentation surgery (Table 2).

\section{How participants look at the breasts of other women}

Most women (32.34\%) preferred type 1 of breast shapes (full on top \& bottom). Only $11.22 \%$ of participants were of the opinion that breast size does not affect female attractiveness and only $12.21 \%$ thought that large breast size is very attractive. When asked about being sexually aroused when seeing beautiful attractive breasts most women $(41.91 \%)$ do not care (Table 3 ).

\section{Husbands' point of view}

A majority of responders $(79.21 \%)$ said that their husbands were satisfied with size and shape of their breasts. Their husbands would not prefer the wives to do any surgery for breast augmentation (88.45\%). Participants would like their husbands to stimulate their 
breasts during foreplay sometimes $(46.86 \%)$ or in all sexual encounters $(30.69 \%)$. The preferred method was both orally and manually $(45.87 \%),(60.29 \%)$ of those who did not like breast stimulation said that the reason was that they would prefer other sites to be stimulated. The ability to reach orgasm by breast stimulation only in all sexual encounters was reported by $(15.51 \%)$ of participants (Table 4).

\section{Breast changes during sex}

An increase in the breast size during intercourse was reported by $(34.66 \%)$ of participants. In the opinion of our participants, sexual attractiveness of breasts did not change either postpartum $(58.42 \%)$ or by getting older $(61.39 \%)$. Only $(16.5 \%)$ of husbands verbally abused their wives for breast changes postpartum (Table 5).

Table 1: Demographic data

\begin{tabular}{lcc}
\hline Variable $(\mathrm{n}=303)$ & $\mathrm{n}$ & $\%$ \\
\hline Age (years) & 10 & 3.3 \\
$<20^{*}$ & 166 & 54.79 \\
$20-29^{*}$ & 109 & 35.97 \\
$30-39^{*}$ & 18 & 5.94 \\
$40-50$ & & \\
& & 8.58 \\
Education level & 26 & 36.96 \\
Can only read and write & 112 & 54.46 \\
Finished secondary school & 165 & \\
Has a university degree & & \\
\hline
\end{tabular}

*child bearing period

Table 2: Participant's breast characteristics

\begin{tabular}{|c|c|c|}
\hline Variable $(n=303)$ & $\mathrm{n}$ & $\%$ \\
\hline Shape & 82 & 27.06 \\
\hline 1. Full on top\& bottom & 56 & 18.48 \\
\hline 2. Wide at the root with pointy nipples & 121 & 39.93 \\
\hline 3. Full on top & 36 & 11.88 \\
\hline 4. Slim at the top, full on the bottom & 7 & 2.31 \\
\hline 5. Root is wide but not full & 1 & 0.33 \\
\hline \multicolumn{3}{|l|}{ 6. Root is narrow and not full } \\
\hline Areolar color & 190 & 62.71 \\
\hline Reddish brown & 111 & 36.63 \\
\hline Dark brown & 1 & 0.33 \\
\hline \multicolumn{3}{|l|}{ Non-responders } \\
\hline Bra size & 52 & 17.16 \\
\hline A 35 (Very small) & 75 & 24.75 \\
\hline B 38(Small) & 91 & 30.03 \\
\hline C 40(Medium) & 65 & 21.45 \\
\hline D 44(Large) & 19 & 6.27 \\
\hline DD 46(X large) & 1 & 0.33 \\
\hline \multicolumn{3}{|l|}{ E 48 (XX large) } \\
\hline \multicolumn{3}{|c|}{ How would you think about large breast } \\
\hline size? & 37 & 12.21 \\
\hline Very attractive & 96 & 31.68 \\
\hline Not attractive & 170 & 56.11 \\
\hline Uncomfortable & & \\
\hline
\end{tabular}


As a teenager, were you satisfied about your breast size and shape?

202

48

49

No

I did not care

Non-responders

Would you like to do surgery for breast augmentation?

Yes, I hope so

No, never

May be

Non-responders

Table 3: Participants' view of breasts of other women

Variable $(n=303)$

Preferred shape

1. Full on top\& bottom

98

30

2. Wide at the root with pointy nipples

3. Full on top

4. Slim at the top, full on the bottom

5. Root is wide but not full

6 . Root is narrow and not full

Does breast size affect

female attractiveness?

Yes, of course

$41 \quad 13.53$

No

How would you think about

large breast size?

Very attractive

Not attractive

Uncomfortable

Do you become sexually aroused when you see beautiful attractive breasts? 
Table 4: Sexual aspects connected to breasts

n

$\%$
Variable $(n=303)$

Is your husband satisfied with size and shape of your breast?

Yes

No

He doesn't comment

Would your husband prefer you to do any surgery for breast augmentation?

Yes

No

Non-responders

Do you like your husband to stimulate your breast during coitus?

Yes, all the times

Sometimes

Never, I hate that

Which method of stimulation would you prefer?

Oral

Manual

Both

Non-responders
240

28

35

31

268

10.23

88.45

1.32

142

68

30.69

46.86

22.44
If you do not like breast stimulation, give a reason?

I prefer other sites of stimulation

Can you reach orgasm by breast stimulation only?

Yes, all times

Never

Non-responders 
Table 5: Factors affecting breast changes

Variable $(n=303)$

$\%$

Do you feel changes of your breasts during sexual activity?

$173 \quad 57.09$

Yes

128

No

0.66

Non-responders

If yes, what changes do you feel with sexual stimulation?*

Increased size

Nipple erection

Increased areola pigmentation

All of the above

Non-responders

Do you think that your breasts are the same sexually attractive as before pregnancy and breast feeding?

Yes

$102 \quad 33.66$

No

Non-responders

Has your husband ever verbally abused you about changes of breast shape or size postpartum?

$\begin{array}{cc}50 & 16.5 \\ 162 & 53.47 \\ 53 & 17.49 \\ 38 & 12.54\end{array}$

No

He likes these changes

Non-responders

With getting old, do you feel that breast attractiveness decrease?

$\begin{array}{cc}103 & 33.99 \\ 186 & 61.39 \\ 14 & 4.62\end{array}$

No

Non-responders

*More than one answer was allowed

\section{DISCUSSION}

Having breasts is a no-win situation for women because this female part of the anatomy has taken on a whole new meaning as a sexualized object and one that continues to be objectified through the mass media. Typically, when one part of a woman's body receives so much attention as the result of male scrutiny, overall body image is negatively impacted. This causes women to obsess, over-evaluate, and become self-conscious about their appearance and breast size. Breasts then take on an identity separate from the body because of media objectification, become highly sexualized, and become psychologically separate from a women's personal being ${ }^{[14]}$.
In general, our participants seemed to be satisfied with their current and past breast size and shape. Bra size (D40) was the most common size used by our participants $(30.03 \%)$ although large and extralarge sizes were also common $(27.72 \%)$. According to a survey by the lingerie retailer "Intimacy", the average bra size in the USA has jumped from a 34B 20 years ago to a 34DD in 2013. Weight gain and breast implants are claimed to be among the reasons ${ }^{[15]}$. Large sized breasts were found uncomfortable by $56.11 \%$ of women in the present study. The magnitude of forces generated by the breast to the thoracic spine ranged between 8.5 pounds of force for bra size 30 to 110 pounds of force for bra size $60^{[16]}$. Large breasts can contribute to numerous negative health outcomes in 
women, including upper limb, neck, back and head pain. These problems have been found to be severe enough to force women with large breasts to seek reduction mammoplasty ${ }^{[17,18]}$.

Interestingly, Guéguenon $^{[19]}$ found that French motorists offered more help for women with larger busts. Alike, men, in a nightclub, approached women with larger breasts more than women with small breasts $^{[20]}$. Moreover, an American study ${ }^{[21]}$ found that waitresses' tips increased with increase in breast size.

Comparing attractiveness of female breasts (among other physical traits) between $(16,686)$ men and $(17,329)$ women, Prantl and Gründl ${ }^{[22]}$ found that a clear difference was found with regard to the ideal bust size, $40 \%$ of men but only $25 \%$ of women preferred a large bust. Male preference of bigger breasts was still found when heterosexual men were compared to non-heterosexual women ${ }^{[23]}$. Such gender difference is consistent with our findings, where only $(11.22 \%)$ of women said that female breast size does not affect female attractiveness and (12.21\%) only said that large breast size is very attractive.

In our research, the majority of women $(32.34 \%)$ preferred "full on top and bottom" breast shape. There has been little discussion in the published literature regarding breast shape preferences by women. Hsia and Thomson ${ }^{[24]}$ conducted a study to ascertain differences in breast shape preferences between plastic surgeons, women seeking breast augmentation, and lay persons. Augmentation patients, compared with plastic surgeons, rated the convex breasts as more attractive and natural and as being closer to their personal ideal breast shape and society's ideal breast shape. Plastic surgeons rated the concave breasts as more attractive, more natural, and closer to their personal ideal. The lay respondents' ratings were generally intermediate between those of the patient and surgeon groups. The similarity between the results of this study and ours indicate that women preferences are very specific and differ from those of doctors (especially males).

In the current study, $15.15 \%$ of women reported the ability to reach orgasm by breast stimulation only in all sexual encounters, which is near to the $12 \%$ reported by Younis et al. ${ }^{[25]}$. Noteworthy, Komisaruk et al. ${ }^{[26]}$ used functional MRI to map sensory cortical responses to clitoral, vaginal, cervical, and nipple selfstimulation whereas,t, unexpectedly, nipple/breast self-stimulation activated not only the 'expected' thoracic sensory homuncular region but also the region of the paracentral lobule that overlaps with the region activated by clitoral, vaginal, or cervical selfstimulation. They stated that 'this finding is consistent with many women's reports that nipple/breast stimulation is erotogenic and can elicit orgasms'.
Breast augmentation is the most commonly performed aesthetic surgical procedure in the USA ${ }^{[27]}$. In 2015, nearly 280,000 American-women and teenagers underwent surgery to have enlarged breasts ${ }^{[28]}$. Almost $80 \%$ of husbands of our participants were satisfied with size and shape of their wives' breasts. Only $10 \%$ of husbands would prefer that their wives do a breast augmentation surgery. Also, $11.55 \%$ of the responders liked to perform a breast augmentation surgery. One cannot exclude economic reasons and the prevailing nature of female clothing of our participants.

Breast changes during intercourse confirm the long established changes described by Masters and Johnson $^{[29]}$. However, breast changes with aging did not decrease attractiveness in the opinion of the majority of our responders $(61.39 \%)$. Aging women move away from their ideals of beauty; specifically, they gain weight which causes body dissatisfaction. However, importance attached to the body decreases with age, which attenuates body dissatisfaction ${ }^{[30]}$. Rauh et al. ${ }^{[31]}$ concluded that factors affecting breast changes would be age at first full-term pregnancy, number of pregnancies, breastfeeding, and BMI of the fathers.

Some of our findings can be of help to the sex therapist. Of them, only $22.44 \%$ of women did not like their husbands to stimulate their breasts during foreplay. On the contrary, Levin and Meston ${ }^{[32]}$ studied the effects of nipple and breast stimulation in sexually experienced 153 women. The major findings in regard to the women were that $(81.5 \%)$ reported that stimulation of their nipples/breasts caused or enhanced their sexual arousal, (78.2\%) agreed that when sexually aroused such manipulation increased their arousal, (59.1\%) had asked to have their nipples stimulated during love making. Exactly how nipple/ breast stimulation influences sexual arousal is poorly understood. Reviewing the endocrinology of human sexual arousal, Bancroft ${ }^{[33]}$, commented on its complexity and on the as yet uncertain role(s) of prolactin and oxytocin. Moreover, experiments on the release of prolactin during nipple/breast stimulation in non-lactating women have given conflicting results. Kolodney et al.$^{[34]}$ reported that it was increased, while a later study ${ }^{[35]}$ did not show any increase in prolactin during either solitary or multiple episodes of nipple stimulation over 24 hours.

\section{Limitations of the study}

Illiterate women were excluded from the study; this eliminated a large sector of women which makes the study less representative of the Egyptian society. Also, cultural difficulty and conservative community limited our ability to pose all needed questions as this would 
have led to a higher rejection rate (rejection rate was $8.2 \%$ ).

\section{CONCLUSION}

The current study outlined importance of oral and manual stimulation of the breasts during foreplay. It was even confirmed that some women can reach orgasm by the mere stimulation of their breasts. Unlike men, women think that large breasts are not only non-attractive but also uncomfortable.

\section{CONFLICT OF INTEREST}

There are no conflicts of interest.

\section{REFERENCES}

1. Kinnunen T. Breast Augmentation Surgery: Carving the Flesh as Female. In: Sex, violence and the body, Burr V. and Hearn J. (Eds). Palgrave Macmillan, London, New York, 2008, P34.

2. Jones, D. An evolutionary perspective on physical attractiveness. Evol Anthropol 1996; 5: 97-109.

3. Furnham A. Swami V. Perception of female buttocks and breast size in profile. Soc Behav Personal 2007; 35(1):1-7.

4. Dixson BJ, Vasey PL, Sagata K, Sibanda N, Linklater WL, Dixson AF. Men's preferences for women's breast morphology in New Zealand, Samoa, and Papua New Guinea. Arch Sex Behav 2011;40(6):12719-.

5. Dixson BJ, Duncan M, Dixson AF. The role of breast size and areolar pigmentation in perceptions of women's sexual attractiveness, reproductive health, sexual maturity, maternal nurturing abilities, and age. Arch Sex Behav 2015;44(6):168595-.

6. Furnham A, Dias M., McClelland A. The role of bodyweight, waist-to-hip ratio, and breast size in judgments of female attractiveness. Sex Roles 1998; 39(3-4): 311-326.

7. Zelazniewicz AM, Pawlowski B. Female breast size attractiveness formen as a function of sociosexual orientation (restricted vs. unrestricted). Arch Sex Behav 2011; 40(6):1129-1135.

8. Gallup,G.G. Permanent breast enlargement in human females: A sociobiological analysis. J Hum Evol 1982;11, 597-601.

9. Symons D. Beauty is the adaptations of the beholder: The evolutionary psychology of human female sexual attractiveness. In Sexual nature, sexual culture
Abramson PR \& Pinkerton SD (Eds.), 1995; Chicago: University of Chicago Press P80.

10. Jasieńska G, Ziomkiewicz A, Ellison PT, Lipson SF, Thune I. Large breasts and narrow waists indicate high reproductive potential in women. Proc Biol Sci 2004 ; Ser. B 271: 1213-1217.

11. Lynn M. Determinants and consequences of female attractiveness and sexiness: realistic tests with restaurant waitresses. Arch Sex Behav 2009;38(5):737- 45 .

12. Prantl L, Gründl M. Males prefer a larger bust size in women than females themselves: An experimental study on female bodily attractiveness with varying weight, bust size, waist width, hip width, and leg length independently. Aesthetic plastic surgery. 2011;35(5):693 -702.

13. Tovée MJ, Cornelissen PL. Female and male perceptions of female physical attractiveness in frontview and profile. Br J Psychol. 2001;92:391- 402.

14. Worrell A. An Examination of Women's Body Image and Sexual Satisfaction Following Breast Augmentation. PhD dissertation, Faculty of the Graduate School of St. Mary's University. ProQuest; 2008, P52.

15. Dicker R. American Bra Size Average Increases From 34B to 34DD In Just 20 Years, Survey Says. Available at: http://www.huffingtonpost.com/201324/07//brasize survey_n_3645267.html Accessed November $10^{\text {th }} 2016$.

16. Hansraj KK. Breast Forces on the Spine. Surg Technol Int 2016;28:311- 5 .

17. Ryan EL. Pectoral girdle myalgia in women: a 5 year study in a clinical setting. Clin J Pain 2000; 16: $298 \mathrm{e} 303$.

18. Greenbaum AR, Heslop T, Morris J, Dunn KW. An investigation of the suitability of bra fit in women referred for reduction mammaplasty. Br J Plast Surg 2003; 56:230e 236 .

19. Guéguen N. Bust size and hitchhiking: a field study. Percept Mot Skills 2007;105:1294- 8.

20. Guéguen N. Women's bust size and men's courtship solicitation. Body Image 2007;4(4):386 -90.

21. Lynn M. Determinants and consequences of female attractiveness and sexiness: realistic tests with restaurant waitresses. Arch Sex Behav 2009; 38(5):737 -45. 
22. Prantl L, Gründl M. Males prefer a larger bust size in women than females themselves: an experimental study on female bodily attractiveness with varying weight, bust size, waist width, hip width, and leg length independently. Aesthetic Plast Surg 2011;35(5):693- 702 .

23. Valentova JV, Bártová K, Štěrbová Z, Varella MA. Influence of sexual orientation, population, homogamy, and imprinting-like effect on preferences and choices for female buttock size, breast size and shape, and WHR. Pers Indiv Differ, 2017 Jan 31;104:313- 9.

24. Hsia HC, Thomson JG. Differences in breast shape preferences between plastic surgeons and patients seeking breast augmentation. Plast Reconstr Surg 2003;112(1):312 -20.

25. Younis I, Fattah M, Maamoun M. Female hot spots: extragenital erogenous zones. Human Andrology 2016;6(1):20- 6 .

26. Komisaruk BR, Wise N, Frangos E, Liu WC, Allen K, Brody S. Women's clitoris, vagina, and cervix mapped on the sensory cortex: fMRI evidence. J Sex Med 2011; 8:2822-2830.

27. Hidalgo DA, Spector JA. Breast augmentation. Plast Reconstr Surg 2014;133(4):567e-83e.

28. The American Society of Plastic Surgeons. 2015 Plastic Surgery Statistics. Available : https://www. plasticsurgery.org/news/plastic-surgery-statistics. Accessed November 19 ${ }^{\text {th }}, 2016$.
29. Masters WH, Johnson VE (1966).Human sexual response ( $1^{\text {st }}$ ed.). Boston: Little, Brown \&Company. Lipowska M, Lipowski M, Olszewski H, DykalskaBieck D. Gender differences in body-esteem among seniors: Beauty and health considerations. Arch Gerontol Geriatr 2016;67:160- 70.

30. Rauh C, Faschingbauer F, Haeberle L, Jud SM, Heusinger K, Fasching PA, Goecke TW, Rajakaruna N, Voigt F, Bani MR, Lux MP, Renner SP, Loehberg CR, Hartmann A, Schulz-Wendtland R, Beckmann MW, Bayer CM. Factors influencing breast changes after pregnancy. Eur J Cancer Prev 2013;22(3):259 -61.

31. Levin R, Meston C. Nipple/Breast stimulation and sexual arousal in young men and women. J Sex Med. 2006;3(3):450- 4.

32. Bancroft J. The endocrinology of sexual arousal. J Endocrinol 2005;86:411-27.

33. Kolodney RC, Jacobs LS, Daughaday WH. Mammary stimulation causes prolactin secretion in non-lactating women. Nature 1972;238:284-6.

34. Ratner RE, Sherry SH, Guay AT. Secretion of prolactin after acute and chronic stimulation of the breast: Effect of timing during the menstrual cycle. Fertil Steril 1982;38:410-4. 